\title{
Doppler Events in the Solar Photosphere: The Coincident Superposition of Fast Granular Flows and p-Mode Coherence Patches
}

\author{
R. Lee McClure ${ }^{1,2}$ - Mark P. Rast ${ }^{1,2}$. \\ Valentin Martínez Pillet ${ }^{3}$
}

Received: 22 October 2018 / Accepted: 7 January 2019 / Published online: 5 February 2019

(C) The Author(s) 2019

\begin{abstract}
Observations of the solar photosphere show spatially compact large-amplitude Doppler velocity events with short lifetimes. In data from the Imaging Magnetograph eXperiment (IMaX) on the first flight of the SUNRISE balloon in 2009, events with velocities in excess of $4 \sigma$ from the mean can be identified in both intergranular downflow lanes and granular upflows. We show that the statistics of such events are consistent with the random superposition of strong convective flows and p-mode coherence patches. Such coincident superposition complicates the identification of acoustic wave sources in the solar photosphere, and may be important in the interpretation of spectral line profiles formed in solar photosphere.
\end{abstract}

Keywords Granulation · Oscillations, Solar · Waves, Acoustic

\section{Introduction}

Spectropolarimetric inversion of data from the Imaging Magnetograph eXperiment (IMaX) instrument (Martínez Pillet et al., 2011) on the first flight of the SUNRISE stratospheric balloon (Solanki et al., 2010) yielded two 20-30 minute high resolution time-series of the photospheric Doppler velocity with 33 second cadence. These time series show compact intermittent flashes of extreme Doppler values in the intergranular lanes and somewhat less conspicuous but complementary outstanding Doppler values within granules (see movie in supplementary material).

Electronic supplementary material The online version of this article (https://doi.org/10.1007/s11207-019-1395-9) contains supplementary material, which is available to authorized users.

R.L. McClure

rachel.mcclure@colorado.edu

1 Department of Astrophysical and Planetary Sciences, University of Colorado, Boulder, CO 80309, USA

2 Laboratory for Atmospheric and Space Physics, University of Colorado, Boulder, CO 80303, USA

3 National Solar Observatory, Boulder, CO 80303, USA 
Two physically distinct processes contribute to the magnitude of the Doppler velocity observed in the solar photosphere. Thermal convection is visible as granulation, with characteristic Doppler velocity amplitudes between $\pm 0.5-1.5 \mathrm{~km} \mathrm{~s}^{-1}$ (Title et al., 1989), and the random superposition of the solar acoustic oscillations (Ulrich, 1970) with maximum individual mode amplitudes of about $\pm 15 \mathrm{~cm} \mathrm{~s}^{-1}$ (e.g., Christensen-Dalsgaard, 2003) producing coherence patches with median vertical velocity amplitude of about $\pm 0.5 \mathrm{~km} \mathrm{~s}^{-1}$ and maximum amplitudes on the order of $\pm 1 \mathrm{~km} \mathrm{~s}^{-1}$ (Leighton, Noyes, and Simon, 1962; Libbrecht, 1988). In this paper we study these two components of the flow at high spatial resolution and show how together they yield the observed Doppler flashes.

In Section 2 we describe the SUNRISE I IMaX data in more detail and discuss the criteria used to identify the extreme Doppler velocities events. In Section 3 we separate granular and p-mode contributions to the Doppler maps, examine the two components individually, and show that the number and amplitude distribution of extreme events are statistically consistent with the random superposition of locally strong granular flows and p-mode coherence patches. Implications are discussed in Section 4.

\section{Observations}

The SUNRISE I stratospheric balloon mission observed the Sun for 130 hours in 2009. The balloon carried a $1 \mathrm{~m}$ Gregorian telescope with an effective focal length of $\sim 25 \mathrm{~m}$ at a float altitude of $35-40 \mathrm{~km}$ (Solanki et al., 2010). The post-focus IMaX instrument used two custom nematic liquid crystal variable retarders, a commercial polarizing beamsplitter, and a double-pass LiNbO3 etalon (Álvarez-Herrero et al., 2006) to measure all four Stokes parameters within the Zeeman-sensitive Fe I $525.02 \mathrm{~nm}$ line and in the neighboring continuum over a $50 \times 50$ arcsecond field of view with a cadence between 10 and $33 \mathrm{~s}$ (Martínez Pillet et al., 2011). We present results from measurements taken at four spectral line positions $(-80,-40,+40$, and $+80 \mathrm{~m} \AA)$ and in the neighboring continuum $(+227 \mathrm{~m} \AA)$. Two regions near solar disk center were observed with spectral resolution of $85 \mathrm{~m} \AA$ at a cadence of 33 seconds, one for a total of 23 minutes and the other for 32 minutes (hereafter called image Set 1 and image Set 2, respectively). A Milne-Eddington inversion (MILOS-Orozco Suárez et al., 2007; 2010) was used to determine the three magnetic field components, lineof-sight velocity, and plasma temperature over the field of view of each image frame in the two time series. Figure 1a displays the line-of-sight velocity in a single frame. Animations of the full time series are available as supplementary material.

The region under consideration is free of any large-scale magnetism (see Figure 1b). Granulation is readily discernible, and acoustic oscillations are apparent as a time-dependent wavering across the field of view in the animated time series and as large regions $(\sim 2 \times$ $2 \mathrm{Mm}^{2}$ patches) of enhanced or depressed vertical velocities in the still image of Figure 1a.

\subsection{Event Definition}

Spatially compact large-amplitude Doppler velocity events with a duration much shorter than the lifetime of granules are observed at apparently random intervals and locations in the image time series. These events occur with both signs; downflow events appear as striking flashes in the intergranular lanes and upflow events appear as high-velocity localized sites within granules. With the color table selected for Figure 1a, the events can be seen as compact bright white regions in the intergranular lanes (e.g., at $(x, y)=(2,24)$ in Figure 1a) and bright yellow areas in the granules $(e . g$., at $(x, y)=(25,15)$ in Figure 1a). 


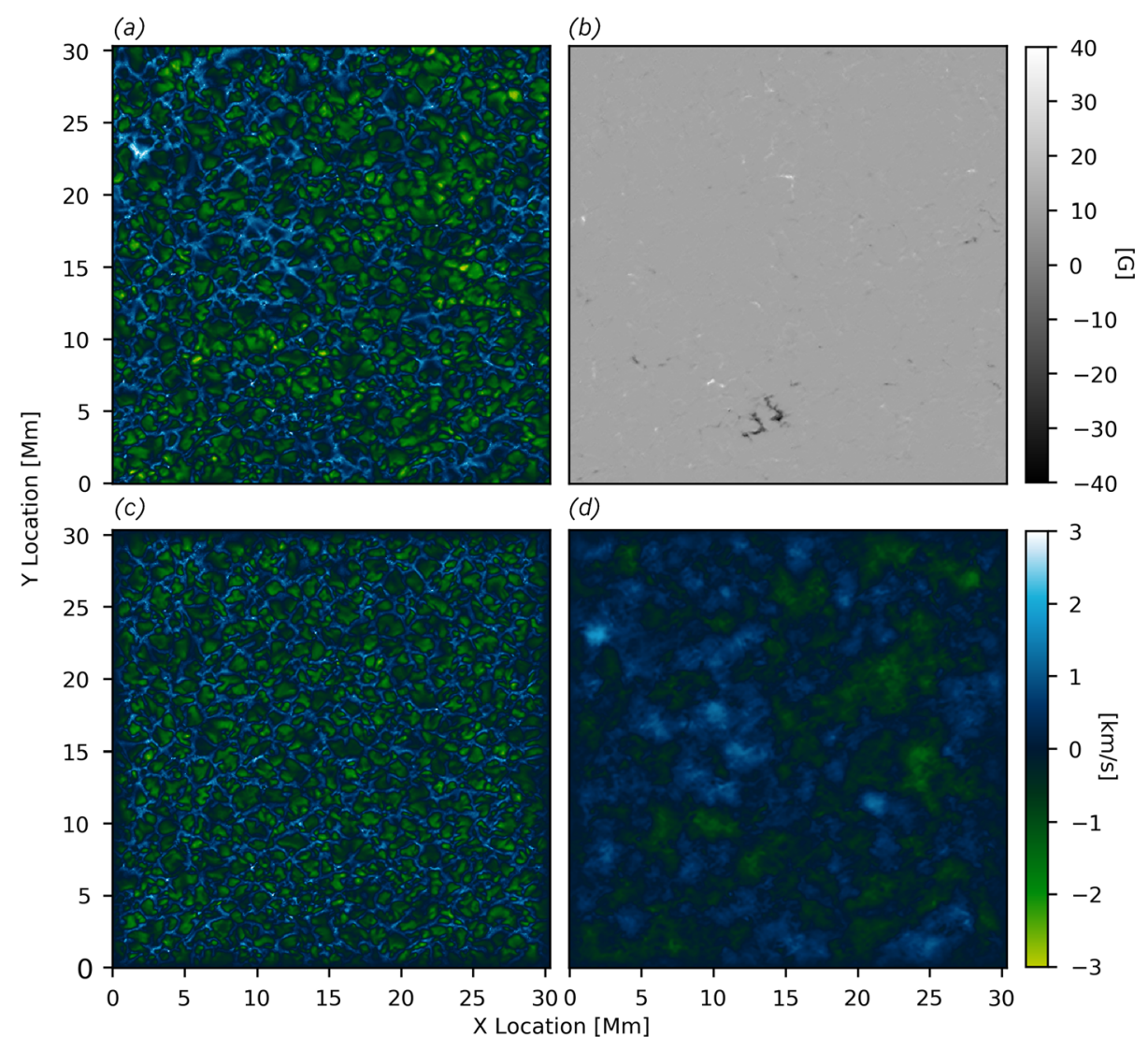

Figure 1 Single frame from the line-of-sight velocity time series a derived from the IMaX spectrograph on the SUNRISE I balloon flight. Positive values correspond to downflows and negative ones to upflows. In c and $\mathbf{d}$, the granulation and p-mode contributions are shown using the same color scaling. Image $\mathbf{b}$ shows the longitudinal magnetic field over the same region with gray scale values ranging from -40 to $40 \mathrm{G}$. The image is saturated to emphasize the weak field.

For analysis, we defined a Doppler event to be any region with an area of at least 10 pixels $^{2}\left(\sim 400 \mathrm{~km}^{2}\right)$ that shows velocities in excess of $4 \sigma$ above or below the temporal and spatial mean over three or more consecutive frames. This corresponds to velocities below -2.65 or above $1.98 \mathrm{~km} \mathrm{~s}^{-1}$ in time-series Set 1 and below -2.71 or above $2.11 \mathrm{~km} \mathrm{~s}^{-1}$ in Set 2. The average event rate across the two time series is $1.9 \pm 0.3$ upflow events and $2.0 \pm 0.3$ downflow events per frame over the $30 \times 30 \mathrm{Mm}^{2}$ central subregion retained after the apodization we employed in the Fourier filter described below.

\subsection{Convective and Acoustic Contributions}

As discussed earlier, there are two contributions to the Doppler velocity measured at any position in the solar photosphere: the convective flow, and the acoustic oscillations. We separated these two contributions by applying a Fourier filter to the Doppler data (e.g., Hill, 1988; Schou et al., 1998). The images were spatially apodized using a two-dimensional cosine-bell taper of width 39 pixels applied to each edge, Fourier transformed in time and 


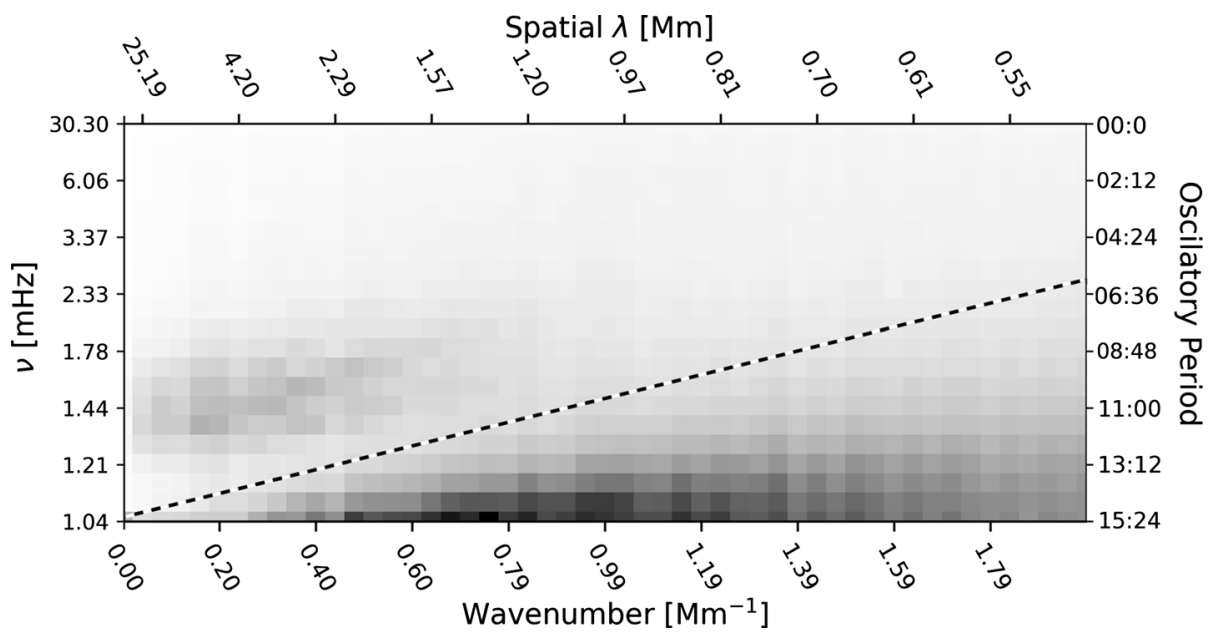

Figure 2 Average power spectrum of the two Doppler-velocity time series. The spectrum was azimuthally averaged in $k$, with annuli widths corresponding to the wavenumber resolution. Application of a $5 \mathrm{~km} \mathrm{~s}^{-1}$ filter (indicated by the dashed line) approximately separates granulation below the line from acoustic modes above the line.

space, and separated into the granular and p-mode components either side of $5 \mathrm{~km} \mathrm{~s}^{-1}$ (see Figure 2). Single frames of the resulting granular and acoustic contributions are shown in Figure $1 \mathrm{c}$ and $1 \mathrm{~d}$.

The distributions of Doppler velocities measured for each component are shown in Figure 3. Not surprisingly, the p-mode contribution is normally distributed about zero, since it results from the random superposition of oscillatory symmetric eigenfunctions. On the other hand, the distribution of the convective velocities is significantly non-Gaussian, with enhanced occurrence of large amplitude flows of both signs. The mean is shifted to negative values because the area of the upflowing regions exceeds that of the downflows, and the distribution is asymmetric, with extreme values more prevalent in downflowing regions (positive values in Figure 3). The median upflow speed $\left(0.60 \mathrm{~km} \mathrm{~s}^{-1}\right.$ for Set 1 and $0.61 \mathrm{~km} \mathrm{~s}^{-1}$ for Set 2) is higher than the median downflow speed $\left(0.43 \mathrm{~km} \mathrm{~s}^{-1}\right.$ for Set 1 and $0.45 \mathrm{~km} \mathrm{~s}^{-1}$ for Set 2), but the upflow speed distribution is much narrower than the downflow speed distribution. While the peak magnitude of downflow speeds $\left(\sim 8 \mathrm{~km} \mathrm{~s}^{-1}\right)$ is higher than the peak magnitude of upflow speeds $\left(\sim 6 \mathrm{~km} \mathrm{~s}^{-1}\right)$, see Figure 3, we note the granular upflows are highly structured with peak upflow speeds in small, compact regions approaching those found in downflow lanes.

\section{Coincident Superposition}

To investigate the origin of the extreme Doppler velocity events identified, we tested the hypothesis that such events are caused by the random superposition of the p-mode and granular contributions. We created synthetic datasets by superimposing the observed p-mode and granulation fields, spatially offsetting the two components and coadding them to form synthetic-image time series. Each frame capturing the p-mode contribution was periodically shifted in increments of 20 pixels 32 times, separately horizontally and vertically and simultaneously in both directions. This process created 102 synthetic observations at each time 


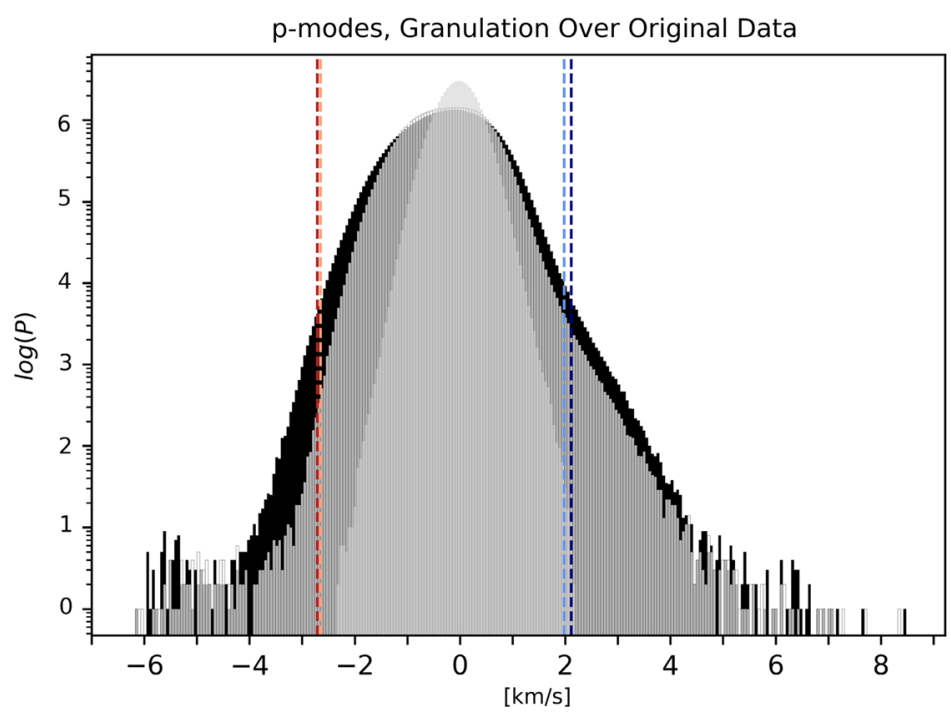

Figure 3 Doppler-velocity distributions, with the p-mode and granulation contributions plotted in light and dark gray, respectively, and the total distribution shown in black. Vertical dashed lines indicate upflow and downflow velocities of magnitudes greater than $4 \sigma$ from their means (Set 1 upflow orange, Set 2 upflow red, Set 1 downflow light blue, Set 2 downflow dark blue). The p-mode contribution is normally distributed about zero, while the granulation shows enhanced high-speed tails of both signs.

Figure 4 Normalized

probability density of Doppler amplitudes (a) in the synthetic datasets produced by random superposition of the p-mode and convective contributions (red) and the observed time series (blue). Normalized cumulative distributions (b) of the Doppler velocities, taken from the upflow (negative value) side, left to right, and from the downflow (positive value) side, right to left, of the observed data in blue and model data in red.
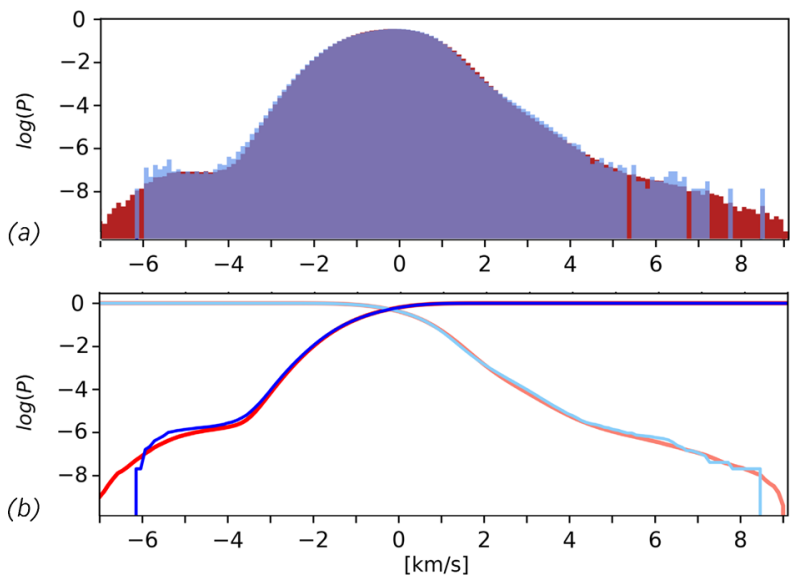

step. Since each frame is over 700 pixels on a side and the minimum event size is taken to be 10 pixels $^{2}$, shifts of 20 pixels avoid double-counting synthetic events.

\subsection{Event Amplitude Distributions}

The model and the original data Doppler amplitude distributions are quite similar (Figure 4), with some small differences apparent in the distribution shoulders and more noise in the observed distribution tails than in the synthetic data for which more time series are available. More constraining than a comparison between the velocity distributions is an assessment of 
Figure 5 Probability density of the number of event counts per frame in the observations and the random superposition model.

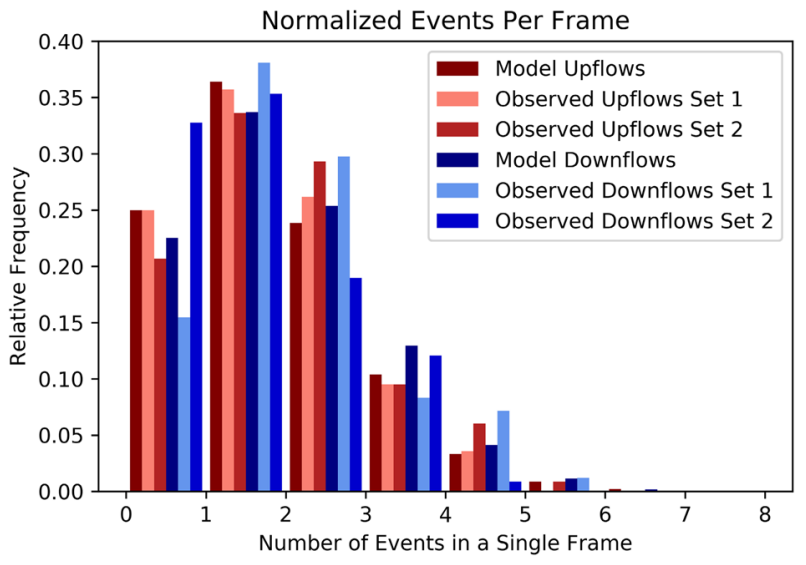

event rates, however, since this captures spatial and temporal coherences within the Doppler map via the event definition.

The overall mean event rates are listed in Table 1 of the Appendix. The observed mean event rate lies within $2 \sigma$ of the mean rate in the synthetic time series produced by the random superposition of granular and p-mode motions. The probability densities of the number of counts per frame (Figure 5) also agrees quite well with that of the observed data, but because of the low number of total counts in the observed data, it is difficult to tell whether any differences are significant.

\subsection{Occurrence Frequency}

To examine this further, we considered the probability of the number of events observed in each frame. Since event counts are small, the distribution approximates a Poisson distribution, so we calculated for each frame the Poisson probability of finding the number of events observed if the mean is that determined from the random superposition model. Figure 6a plots that probability as a function of frame number for the observed time series, along with the distributions of those values for upflow and downflow events in both simulations projected along the $y$-axis. Mean Poisson probability values are indicated with solid horizontal fiducial lines. Similarly, in Figure $6 \mathrm{~b}$ we plot the distribution of the Poisson probability of finding the specific number of events in each frame of a synthetic dataset given the mean number of event counts from all the other model sets. In this way, each model time series is treated in the same manner as the observational time series.

The mean values of the Poisson probabilities of the observed counts (indicated in Figure $6 \mathrm{~b}$ by solid vertical fiducial lines) falls at or within $1 \sigma$ of the means of the synthetic data Poisson probability distributions. This is true for both upflows and downflows in all observational time series. We conclude that the number of outstanding Doppler events in each frame of the observational data is statistically consistent with counts that would be achieved by the random superposition of p-mode and the granular flows.

\section{Conclusion}

The random superposition of solar acoustic oscillation coherence and underlying convective flows can cause intermittent Doppler signals that have amplitudes in excess of $4 \sigma$ from 
(a)

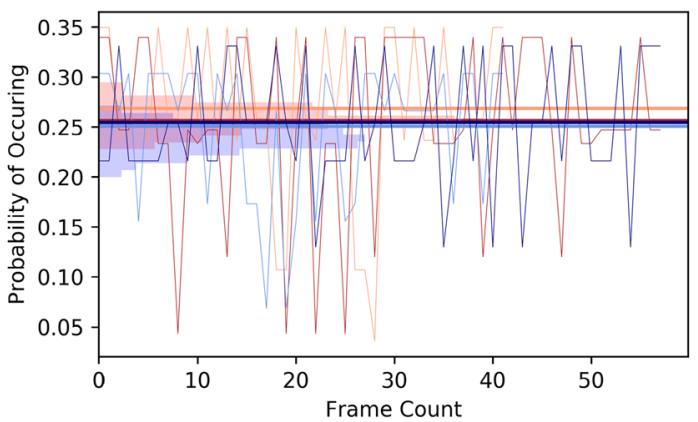

Set 1 Upflows

$\mu=0.27 \pm 0.01$

Set 2 Upflows

$\mu=0.26 \pm 0.01$

Set 1 Downflows

$\mu=0.25 \pm 0.01$

Set 2 Downflows

$\mu=0.25 \pm 0.01$

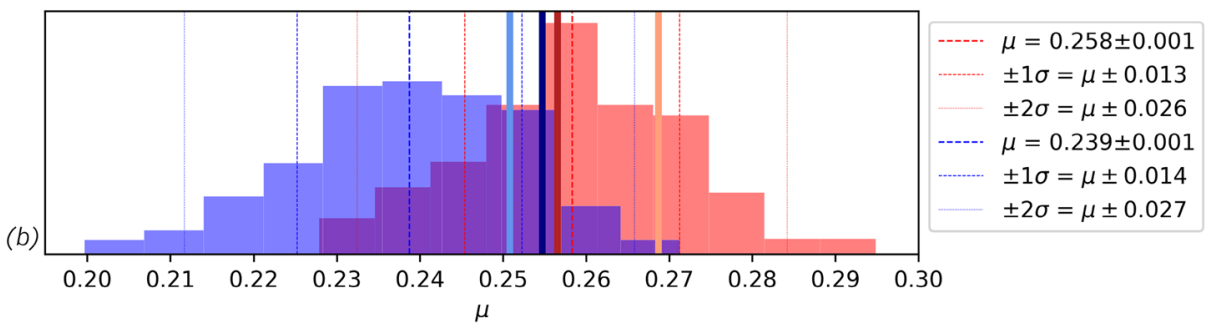

Figure 6 Poisson probability of observing the number of events found in each Doppler-velocity image. In panel a we show the probability as a function of frame number for each observed time series, and the distribution of those probabilities for upflow and downflow events in both simulations projected along the $y$-axis. In panel $\mathbf{b}$ we plot the distribution of the Poisson probability in upflows and downflows of the synthetic model data. The mean Poisson probability values in the observations are indicated with solid fiducial lines in both panels. The mean, $\pm 1 \sigma$, and $\pm 2 \sigma$ Poisson probability values of the model distributions are indicated with vertical dashed and dotted lines in panel $\mathbf{b}$.

the mean, values that are thus faster than $99.7 \%$ of those observed if the distribution is approximated as Gaussian. These Doppler events occur in both upflowing and downflowing regions, and flows of this magnitude are three times more likely to occur in an unfiltered time series than in one from which the p-modes have been removed. It is important to note that these events are real physical occurrences, not an artifact of the observations. Their existence may be critical to the interpretation of spectral lines, the identification of solar acoustic sources, and the understanding of jet-like structures. They may also cause confusion in interpreting observations as instances of specific dynamical mechanisms, particularly when observational time series are too short to adequately remove the p-mode contributions.

Acknowledgements This work was supported in part by the National Science Foundation under grant No. 1616538. The National Solar Observatory is operated by the Association of Universities for Research in Astronomy under a cooperative agreement with the National Science Foundation.

Disclosure of Potential Conflicts of Interest The authors declare that they have no conflicts of interest.

Publisher's Note Springer Nature remains neutral with regard to jurisdictional claims in published maps and institutional affiliations.

Open Access This article is distributed under the terms of the Creative Commons Attribution 4.0 International License (http://creativecommons.org/licenses/by/4.0/), which permits unrestricted use, distribution, and reproduction in any medium, provided you give appropriate credit to the original author(s) and the source, provide a link to the Creative Commons license, and indicate if changes were made. 


\section{Appendix}

Here we tabulate (Table 1) the observed and synthetic event counts, showing that the event statistics are consistent with the random superposition of acoustic mode coherence patches and strong granular flows. Comparable event rates are found in upflows and downflows. This is likely a consequence of being able to resolve the upflow substructure at SUNRISE resolutions.

Table 1 Event counts and event rates for observed data, granular and p-mode components, and the superposition model sets. There are a total of 42 image frames in Set 1 and 58 image frames in Set 2 .

\begin{tabular}{llll}
\hline Data component & & Number of events & $\begin{array}{l}\text { Mean event rate } \\
\left(\mathrm{s}^{-1} \mathrm{Mm}^{-2}\right)\end{array}$ \\
\hline Observed data & & & \\
Upflows & Set 1 & 74 & $5.6 \times 10^{-5} \pm 9 \%$ \\
& Set 2 & 123 & $6.8 \times 10^{-5} \pm 7 \%$ \\
Downflows & Set 1 & 104 & $5.0 \times 10^{-5} \pm 10 \%$ \\
& Set 2 & 99 & $3.6 \times 10^{-5} \pm 10 \%$ \\
Apodized data & & & $5.1 \times 10^{-5} \pm 10 \%$ \\
Upflows & Set 1 & 55 & $5.8 \times 10^{-5} \pm 10 \%$ \\
& Set 2 & 86 & $6.2 \times 10^{-5} \pm 10 \%$ \\
Downflows & Set 1 & 66 & $4.4 \times 10^{-5} \pm 10 \%$
\end{tabular}

Granular component

\begin{tabular}{llll} 
Upflows & Set 1 & 4 & $3.7 \times 10^{-6} \pm 60 \%$ \\
\multirow{2}{*}{ Downflows } & Set 2 & 3 & $2.4 \times 10^{-6} \pm 60 \%$ \\
& Set 1 & 22 & $2.1 \times 10^{-5} \pm 20 \%$ \\
& Set 2 & 14 & $9.8 \times 10^{-6} \pm 30 \%$
\end{tabular}

p-Mode Component

\begin{tabular}{llll} 
Upflows & Set 1 & 0 & 0 \\
\multirow{2}{*}{ Downflows } & Set 2 & 0 & 0 \\
& Set 1 & 0 & 0 \\
& Set 2 & 0 & 0
\end{tabular}

Model

\begin{tabular}{llll} 
Upflows & Set 1 & $1.6 \pm .1 /$ frame & $5.30 \times 10^{-5} \pm 1 \%$ \\
\multirow{2}{*}{ Downflows } & Set 2 & $1.7 \pm .1 /$ frame & $5.70 \times 10^{-5} \pm 1 \%$ \\
& Set 1 & $2.0 \pm .2 /$ frame & $6.87 \times 10^{-5} \pm 1 \%$ \\
& Set 2 & $1.8 \pm .1 /$ frame & $6.00 \times 10^{-5} \pm 1 \%$ \\
\hline
\end{tabular}

\section{References}

Álvarez-Herrero, A., Belenguer, T., Pastor, C., Heredero, R.L., Ramos, G., Martínez Pillet, V., Bonet Navarro, J.A.: 2006, Lithium niobate Fabry-Perot etalons in double-pass configuration for spectral filtering in the visible imager magnetograph IMaX for the SUNRISE mission. In: Society of Photo-Optical Instrumentation Engineers (SPIE) Conference Series, Proc. SPIE 6265, 62652G. DOI. ADS. 
Christensen-Dalsgaard, J.: 2003, Lecture Notes on Stellar Oscillations, fifth edn. Aarhus Universitet Teoretisk Astrofysik Center, Danmarks Grundforskningsfond, http://users-phys.au.dk/jcd/oscilnotes/ print-chap-full.pdf.

Hill, F.: 1988, Rings and trumpets - three-dimensional power spectra of solar oscillations. Astrophys. J. 333, 996. DOI. ADS.

Leighton, R.B., Noyes, R.W., Simon, G.W.: 1962, Velocity fields in the solar atmosphere. I. Preliminary report. Astrophys. J. 135, 474. DOI. ADS.

Libbrecht, K.G.: 1988, Solar and stellar seismology. Space Sci. Rev. 47, 275. DOI. ADS.

Martínez Pillet, V., Del Toro Iniesta, J.C., Álvarez-Herrero, A., Domingo, V., Bonet, J.A., González Fernández, L., et al.: 2011, The Imaging Magnetograph eXperiment (IMaX) for the Sunrise Balloon-Borne Solar Observatory. Solar Phys. 268, 57. DOI. ADS.

Orozco Suárez, D., Bellot Rubio, L.R., Del Toro Iniesta, J.C., Tsuneta, S., Lites, B., Ichimoto, et al.: 2007 , Strategy for the inversion of Hinode spectropolarimetric measurements in the quiet Sun. Publ. Astron. Soc. Japan 59, S837. DOI. ADS.

Orozco Suárez, D., Bellot Rubio, L.R., Martínez Pillet, V., Bonet, J.A., Vargas Domínguez, S., Del Toro Iniesta, J.C.: 2010, Retrieval of solar magnetic fields from high-spatial resolution filtergraph data: the imaging magnetograph experiment (IMaXi). Astron. Astrophys. 522, A101. DOI. ADS.

Schou, J., Antia, H.M., Basu, S., Bogart, R.S., Bush, R.I., Chitre, S.M., et al.: 1998, Helioseismic studies of differential rotation in the solar envelope by the solar oscillations investigation using the Michelson Doppler imager. Astrophys. J. 505, 390. DOI. ADS.

Solanki, S.K., Barthol, P., Danilovic, S., Feller, A., Gandorfer, A., Hirzberger, J., et al.: 2010, SUNRISE: instrument, mission, data, and first results. Astrophys. J. Lett. 723, L127. DOI. ADS.

Title, A.M., Tarbell, T.D., Topka, K.P., Ferguson, S.H., Shine, R.A., SOUP Team: 1989, Statistical properties of solar granulation derived from the SOUP instrument on Spacelab 2. Astrophys. J. 336, 475. DOI. ADS.

Ulrich, R.K.: 1970, The five-minute oscillations on the solar surface. Astrophys. J. 162, 993. DOI. ADS. 